\title{
RESPOSTAS FISIOLÓGICAS ASSOCIADAS A VARIAÇÕES TÉRMICAS DIAGNOSTICADAS POR TERMOGRAMA INFRAVERMELHO EM EQUINOS SUBMETIDOS A ESFORÇO FÍSICO INTENSO
}

Lílian Kátia Ximenes Silva'; Lucieta Guerreiro Martorano²; Welligton Conceição da Silva ${ }^{3}$; Alessandra Belo Reis ${ }^{4}$; Filipe Prudente da Silva ${ }^{5}$; Gleumadson Borges Fernandes ${ }^{6}$; Kedson Alessandri Lobo Neves ${ }^{7}$.

\footnotetext{
${ }^{1}$ Universidade da Amazônia, Santarém, Pará, Brasil, silva_lilian@yahoo.com.br 2 Embrapa Amazônia Oriental, Santarém, Pará, Brasil, lucieta.martorano@embrapa.br

${ }^{3}$ Universidade da Amazônia, Santarém, Pará, Brasil, welligton.flamengo@hotmail.com

${ }^{4}$ Universidade da Amazônia, Santarém, Pará, Brasil, alessandra.belo.reis@gmail.com

${ }^{5}$ Universidade da Amazônia, Santarém, Pará, Brasil, prudentefilipe@gmail.com

${ }^{6}$ Universidade da Amazônia, Santarém, Pará, Brasil, gleumadson@hotmail.com

7 Universidade Federal do Oeste do Pará, Santarém, Pará, Brasil, kedson_neves@hotmail.com
}

RESUMO: Objetivou-se utilizar a termografia infravermelho para avaliar respostas fisiológicas em equinos submetidos a esforço físico intenso. O estudo foi conduzido em Santarém, Pará, utilizando-se 4 (quatro) equinos adultos. Foi realizado o imageamento da região da cabeça e da garupa com termógrafo infravermelho antes e após o treino, o monitoramento da frequência cardíaca $\left(F C ;\right.$ bat $\left.\min ^{-1}\right)$ e frequência respiratória (FR; mov $\left.\mathrm{min}^{-1}\right)$; e, calculou-se o Índice de Conforto (IC) e o Índice de Temperatura e Umidade (ITU). Foram analisados dados médios, extremos e amplitude térmica, avaliando-se a correlação com as variáveis fisiológicas. Na temperatura de superfície da cabeça e garupa antes e após o treino não houve diferença $(P>0,05)$, entretanto foi observada $(P<0,01)$ na amplitude térmica na região da cabeça no padrão branco, sendo superior após o treino e na garupa nos padrões branco e amarelo, indicando que essa variável foi sensível para detectar diferenças térmicas após $\mathrm{O}$ treino. A FR e a FC apresentaram-se elevadas após o treino $(P<0,05)$. A amplitude térmica responde a frequência respiratória de equinos e pode ser descrita por um modelo polinomial com $70 \%$ de confiança, indicando que os animais com amplitude térmica superior a $1,2^{\circ} \mathrm{C}$ já se encontram ofegantes. A termografia infravermelho é recomendada para detectar níveis de estresse térmico em equinos submetidos a esforços intensos.

PALAVRAS-CHAVE: Amplitude térmica, Equinos, Termografia infravermelho. 


\title{
PHYSIOLOGICAL RESPONSES ASSOCIATED TO THERMAL VARIATIONS DIAGNOSED BY INFRARED THERMOGRAM IN EQUINES SUBMITTED TO INTENSE PHYSICAL EFFORT
}

\begin{abstract}
The objective was to use infrared thermography to evaluate physiological responses in equines submitted to intense physical effort. The study was conducted in Santarém (020 26 '35 "S and 54 42' 30" W), in Pará, using four (4) adult equines. We performed the imaging of the head and croup with infrared thermograph before and after training. The heart rate (HR; beat. $\left.\mathrm{min}^{-1}\right)$ and respiratory rate $\left(\mathrm{RR} ; \mathrm{mov}\right.$. $\left.\mathrm{min}^{-1}\right)$ were monitored. The Comfort Index $(\mathrm{Cl})$ and the Temperature and Humidity Index (THI) were calculated. The analysis of variance and Tukey's test were performed, with a significance level of 5\%. Mean, extreme and thermal amplitude data were analyzed and the correlation with the physiological variables was evaluated. We observed a THI of 79.2, indicating a challenging thermal environment for the animals. There was no significant difference $(P>0.05)$ in the surface temperature of the head and croup before and after training. However, we observed difference $(P<0.01)$ in the thermal amplitude in the head region in white pattern, being higher after training and in the rump in white and yellow patterns, indicating that this variable was sensitive to detect thermal differences after training. The HR and RR were elevated after training ( $P$ $<0.05)$. The thermal amplitude responds to the respiratory frequency of equines and can be described by a polynomial model with $70 \%$ confidence, indicating that animals with thermal amplitude higher than $1.2^{\circ} \mathrm{C}$ are already panting. We conclude that infrared thermography is recommended to detect levels of thermal stress in equines subjected to intense stress.
\end{abstract}

KEYWORDS: Equines, Infrared thermography, Thermal amplitude.

\section{RESPUESTAS FISIOLÓGICAS ASOCIADAS A VARIACIONES TÉRMICAS DIAGNOSTICADAS POR TERMOGRAMA INFRARROJO EN EQUINOS SOMETIDOS A ESFUERZO FÍSICO INTENSO}

RESUMEN: Se objetivó utilizar la termografía infrarroja para evaluar respuestas fisiológicas en equinos sometidos a esfuerzo físico intenso. El estudio fue conducido

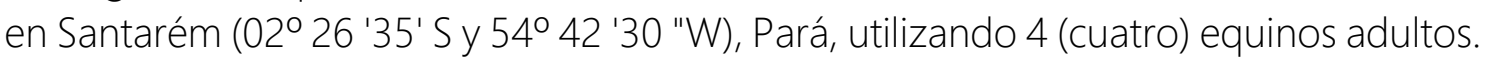
Se realizó la imagen de la región de la cabeza y de la grupa con termógrafo infrarrojo antes y después del entrenamiento. Se monitorizó la frecuencia cardíaca (FC, bat.min ${ }^{1}$ ) y la frecuencia respiratoria $\left(F R\right.$, mov. $\left.\mathrm{min}^{-1}\right)$. Se calculó el Índice de Confort (IC) y el Índice de Temperatura y humedad (ITH). Se realizó análisis de varianza y aplicación del test de Tukey, con un nivel de significancia de 5\%. Se analizaron datos medios, extremos y amplitud térmica, evaluando la correlación con las variables fisiológicas. 
Se observó ITH de 79,2, indicando ambiente térmico desafiante a los animales. En la temperatura de superficie de la cabeza y de la grupa antes y después del entrenamiento no hubo diferencia significativa $(P>0,05)$. Sin embargo, fue observada diferencia $(P<0,01)$ en la amplitud térmica en la región de la cabeza en el patrón blanco, siendo superior después del entrenamiento y en la grupa en los patrones blanco y amarillo, indicando que esa variable fue sensible para detectar diferencias térmicas después del entrenamiento. La FR y la FC se mostraron elevadas después del entrenamiento $(P<0,05)$. La amplitud térmica responde a la frecuencia respiratoria de los equinos y puede ser descrita por un modelo polinomial con un $70 \%$ de confianza, indicando que los animales con amplitud térmica superior a $1,2^{\circ} \mathrm{C}$ ya se encuentran jadeantes. Se concluye que la termografía infrarroja es recomendada para detectar niveles de estrés térmico en equinos sometidos a esfuerzos intensos.

PALABRAS CLAVE: Amplitud térmica, Equinos, Termografía infrarroja.

Os equinos são animais capazes de percorrer curtas ou longas distâncias em alta ou baixa velocidade, respectivamente, com baixo gasto energético (WILSON; WELLER, 2011), os quais são submetidos rotineiramente a esforços físicos excessivos, principalmente em treinamentos, transporte de cargas ou provas esportivas. O exercício físico culmina com a produção de calor, sendo que em torno de $20 \%$ da energia química proveniente da oxidação dos nutrientes transforma-se em energia mecânica, responsável pelo movimento, e o restante (cerca de 80\%) é convertido em energia térmica. Sob condição de esforço físico ocorre acúmulo de energia térmica, o que eleva a temperatura corporal, podendo culminar com uma situação de desconforto térmico animal (CARVALHO; MARA, 2010).

O desconforto térmico aciona mecanismos termorregulatórios, como a sudorese e a vasodilatação periférica, que resulta em redução da pressão arterial, compensada pelo aumento da frequência cardíaca (FC) e, posteriormente, ocorre a elevação da frequência respiratória (FR), conforme Aggarwal e Singh (2008) e Marai e Haeeb (2010). Esses mecanismos termorregulatórios visam à dissipação 
do calor, que em equinos, possuem como vias mais eficientes a evaporação (sudação e ofego), a condução (resfriamento) e a convecção (vasodilatação). A vasodilatação periférica eleva a temperatura da superfície animal e facilita a troca de calor do animal com o ambiente, sendo que a eficácia deste mecanismo depende do gradiente térmica entre a temperatura do corpo animal e das condições térmicas atmosféricas do local (SOUZA et al., 2008).

Fatores relacionados a esforços físicos nesses animais necessitam de maiores investigações científicas para detectar condições limites, principalmente em práticas esportivas, comumente utilizadas em equinos que respondem as variáveis climáticas locais em ocasiões de esforços excessivos (MCCUTCHEON; GEOR, 2008). Como a região Amazônica apresenta em média temperatura, umidade relativa do ar, precipitação pluvial e radiação solar elevado, essas são condicionantes que devem ser consideradas quando os animais são expostos a esforços físicos.
Neste contexto, a termografia infravermelho, sendo um método rápido, preciso e não invasivo é capaz de detectar variações mínimas na temperatura de superfície e seus gradientes (SYKES et al., 2012; MENEGASSI et al. 2015). As imagens termográficas auxiliam na compreensão da termorregulação corporal em razão das mudanças na temperatura superficial e do impacto das condições ambientais sobre o conforto térmico animal (BOUZIDA et al., 2009; ZOTTI, 2010; BARROS et al., 2016). Neste trabalho o objetivo foi avaliar padrões térmicos capazes de explicar respostas fisiológicas em equinos submetidos a esforço físico intenso.

O estudo foi realizado no município de Santarém, Pará, Brasil (020 26' 35" S e $54^{\circ} 42^{\prime} 30^{\prime \prime}$ W). A área de estudo enquadra-se nas características de clima tropical chuvoso cujas temperaturas médias mensais são sempre superiores a $18^{\circ} \mathrm{C}$ cuja na tipologia climática é $\mathrm{Am}_{4}$ (MARTORANO et al., 1993), ou seja, as característica são típicas de clima de monção, com moderada estação seca e 
ocorrência de precipitação média mensal inferior a $60 \mathrm{~mm}$, transicional ente as tipologias Af e Aw, incluída na faixa de totais pluviais anuais entre 1500 mm a 2000 mm (MARTORANO et al., 2017). Foram utilizados 4 (quatro) equinos adultos com cerca de 7,6 $\pm 2,6$ anos de idade, da raça Quarto de Milha e clinicamente saudáveis.

As variáveis meteorológicas (temperatura e umidade relativa do ar, radiação solar e precipitação pluvial) são referentes ao dia 15/09/2017, obtidas da estação meteorológica automática do Instituto Nacional de Meteorologia (INMET), localizada em Santarém (Latitude em UTM -2.5025720S e Longitude $\left.-54.720279^{\circ} \mathrm{W}\right)$. Os valores correspondentes às condições de tempo nesse dia estão expressos na Tabela 1.

Tabela 1. Valores médios das variáveis climáticas registrados em estação meteorológica automática durante o experimento, Santarém, Pará, Brasil.

\begin{tabular}{lc}
\hline Variável Climática & \\
\hline Temperatura do Ar Máxima $\left({ }^{\circ} \mathrm{C}\right)$ & 28.2 \\
Temperatura do Ar Miníma $\left({ }^{\circ} \mathrm{C}\right)$ & 27.3 \\
Temperatura do Ar Média $\left({ }^{\circ} \mathrm{C}\right)$ & 27.8 \\
Umidade Relativa Máxima $(\%)$ & 81.2 \\
Umidade Relativa Miníma $(\%)$ & 76.8 \\
Umidade Relativa Média $(\%)$ & 79.1 \\
Radiação solar global $\left(\mathrm{kJ} / \mathrm{m}^{2}\right)$ & 919.4 \\
Precipitação pluvial $(\mathrm{mm})$ & 0.0 \\
\hline
\end{tabular}

A partir dos valores das variáveis meteorológicas foi calculado o Índice de Temperatura e Umidade (ITU), considerando-se a temperatura e a umidade relativa do ar. Conforme
Azevedo (2005) esse é um índice de referência na avaliação do conforto térmico animal. O ITU foi calculado a partir da equação 1 (THOM, 1959). 


$$
\left.I T U=(0,8 \bar{T})+\left(\frac{U r}{100}\right) *[\overline{(T}-14,4)+46,4\right]
$$

em que $\bar{T}$ é a temperatura média do ar $\left({ }^{\circ} \mathrm{C}\right)$ e Ur é a umidade relativa do ar (\%).

Outro Índice de Conforto (IC) foi calculado, com o intuito de verificar se o ambiente térmico foi estressante ou não para os equinos. $O \quad I C$ foi determinado a partir da equação 2 (JONES, 2009).

$$
I C=\bar{T}\left({ }^{\mathrm{o}} F\right)+U r(\%)
$$

em que $\bar{T}$ é a temperatura média do ar ( $\left.{ }^{\circ} \mathrm{F}\right)$ e Ur é a umidade relativa do ar (\%).
Foram monitoradas a frequência cardíaca (FC; bat $\mathrm{min}^{-1}$ ) e a frequência respiratória $\left(F R ; \operatorname{mov} \min ^{-1}\right)$. A FC foi obtida pela contagem dos batimentos cardíacos com a utilização de estetoscópio manual, e a FR foi medida pela contagem dos movimentos do flanco do animal, ambas mensuradas durante um minuto.

Foram realizadas capturas de imagens termográficas dos animais em dois momentos (antes e após o exercício), considerando-se a Temperatura de Superfície (TS) da região da cabeça e da garupa, entre às 19h00min e 23h30min. Durante o exercício os animais percorreram inicialmente em alta velocidade um trajeto de 90 m, e, em seguida, 50 m para desaceleração dos mesmos, percorrendo ao todo $140 \mathrm{~m}$.

Os dados foram coletados usando termógrafo de infravermelho (FLIR T650sc, Wilsonville, OR, EUA, 2015). Na Figura 1 é possível observar imagens fotográficas durante o processo de obtenção de dados com a câmera termográfica. A câmara possui lente fixa de $25 \mathrm{~mm}$, escala de temperatura de 40 a $150{ }^{\circ} \mathrm{C}$, sensibilidade térmica de $50 \mathrm{mK} \quad\left(<0,05{ }^{\circ} \mathrm{C}\right.$ à temperatura ambiente de $30^{\circ} \mathrm{C}$ ), escala espectral de 
abrangência vai de 0,7 a 100um, mas os alvos imageados apresentam resposta entre 0,7 a 3,0 $\mu$ m e resolução óptica de 640x480 pixels com índice de emissividade máximo de 0,95.

Figura 1. Campanha de campo para captura de imagens termográficas.

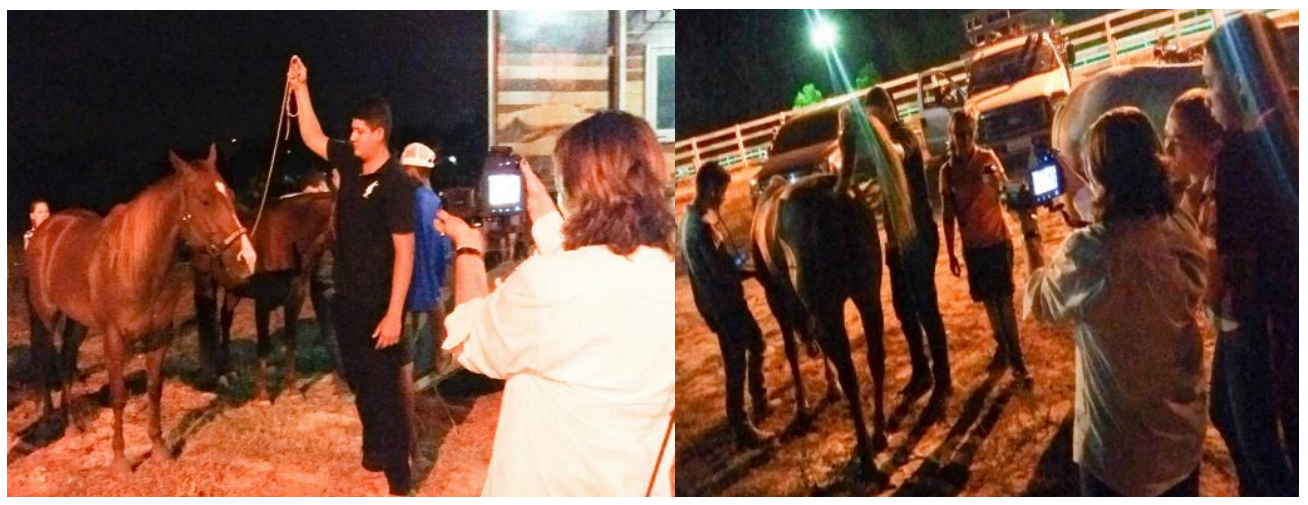

Os termogramas gerados foram posteriormente analisados no programa Flir Tools, 6.3v (FLIR Systems, Inc., Wilsonville, OR, EUA, 2015). Para a obtenção das TS das regiões avaliadas foram adotados os seguintes padrões térmicos em ordem decrescente, identificados pelas cores: branco, vermelho e amarelo. Em cada imagem tratada foram traçados 4 pontos para cada padrão de cor, conforme observado na Figura 2.
Foi verificado se os dados obdeciam um padrão normal de distribuiç̧ão e se havia homogeneidade de variações térmicas. Foram realizadas análises de variância, média e moda, com aplicação do teste de Tukey. O nível de significância adotado foi de 5\%. A estatística descritiva e a comparação das médias foram realizadas usando o software BioEstat, versão 5.3 (AYRES et al., 2007) para identificar padrões com destaques entre as respostas térmicas obtidas das regiões avaliadas. 
Figura 2. Tratamento de imagens termográficas na ferramenta Flir Tools.

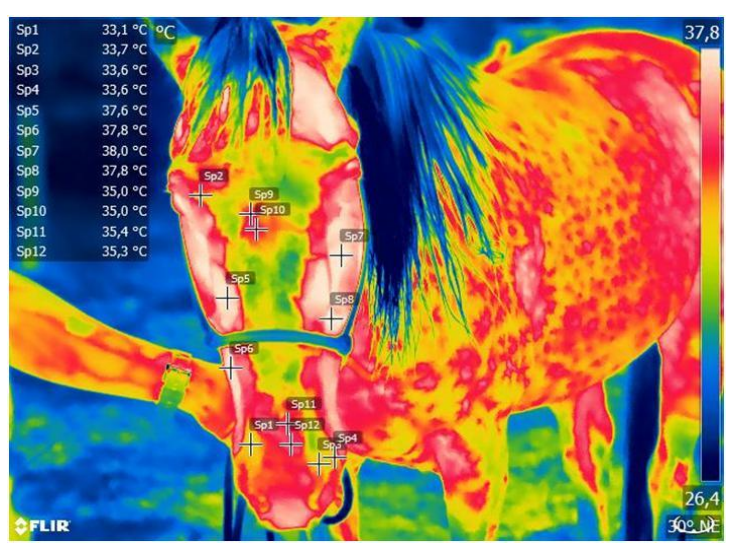

O ITU de 79,2 obtido no estudo durante o experimento aponta um ambiente considerado desafiador para os animais, pois de acordo com Baêta e Souza (1997) valores de ITU até 70 indicam ambiente não estressante, entre 71 e 78 crítico, entre 79 e 83 perigoso e acima de 83, condição de emergência. Entretanto, com base no IC de 109,8 observado, os animais não estavam em ambiente de desconforto térmico, o que segundo Jones (2009) ocorreria com valor acima de 130 quando a dissipação de calor está prejudicada.

Em relação às médias obtidas para as TS da cabeça e garupa antes a após o treino em todos os padrões avaliados não observou-se diferenças $(P>0,05)$

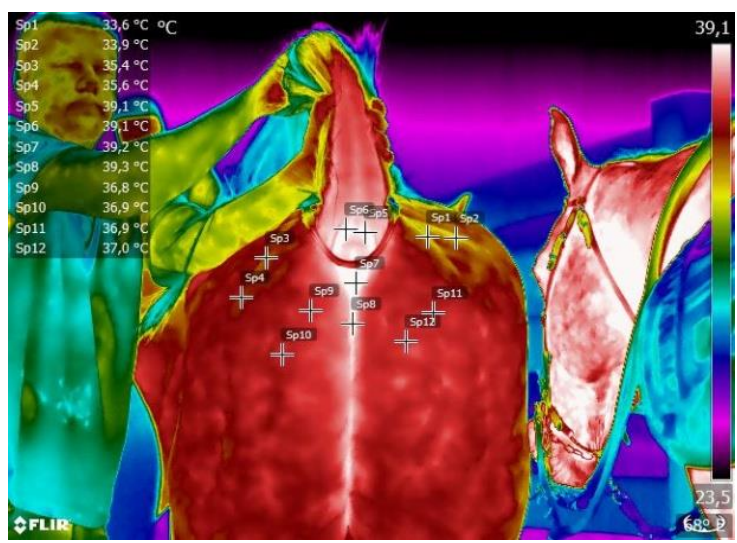

(Tabela 2), concordando com Moura et al. (2011) que mensurando temperaturas de superfície (garupa e peito) não observaram mudanças antes e após o treino, o que pode estar relacionado a baixa capacidade vasomotora de algumas regiões do corpo.

Entretanto, notou-se uma resposta termográfica muito interessante em relação à amplitude térmica das regiões avaliadas. Na cabeça foi observada alteração $(P<0.01)$ no padrão que apresentou as temperaturas mais elevadas (branco), sendo superior após o treino (Tabela 3; Figura 3). Essa maior amplitude térmica observada após o treino na cabeça 
pode ser atribuída a uma ampla irrigação e redistribuição sanguínea nesta região, resultando em aumento no fluxo sanguíneo periférico. A elevação do fluxo sanguíneo do núcleo central para as regiões periféricas do corpo, na tentativa de eliminar o calor produzido, atendendo dessa forma às necessidades termorregulatórias, contribui para elevação da TS (SILVA et al., 2011), podendo mínimas variações de temperatura somente ser observadas pela análise da amplitude térmica.

Tabela 2. Média e desvio padrão das temperaturas de superfície da cabeça e garupa dos equinos antes e após o treino.

\begin{tabular}{lcc}
\hline \multicolumn{1}{c}{ Região/Padrão } & \multicolumn{2}{c}{ Temperatura Média $\left.{ }^{\circ} \mathrm{C}\right)$} \\
\hline Cabeça/Branco & Antes do Treino & Após o Treino \\
Cabeça/Vermelho & $36,2 \pm 3,5$ & $38,5 \pm 1,0$ \\
Cabeça/Amarelo & $36,0 \pm 0,8$ & $36,5 \pm 0,7$ \\
Traseira/Branco & $33,6 \pm 0,8$ & $32,1 \pm 0,6$ \\
Traseira/Vermelho & $39,6 \pm 0,1$ & $39,5 \pm 0,5$ \\
Traseira/Amarelo & $37,7 \pm 0,1$ & $37,5 \pm 0,5$ \\
\hline
\end{tabular}

ab Diferentes letras na mesma linha indicam diferença estatística $(P<0,05)$.

Em relação à garupa constatou-se diferença estatística $(P<0.01)$ nos padrões de temperaturas extremas (branco e amarelo), com valores superiores para amplitude térmica antes do treino (Tabela 2; Figura 4), o que pode estar relacionado à maior concentração de massa muscular nessa região. Marlin et al. (1998) afirmaram que no término do exercício ocorre redução abrupta da demanda de oxigênio (sanguínea) por parte do tecido muscular e da irrigação profusa. Com a necessidade menor de perfusão grande parte do sangue acumulado nos músculos é desviada para a circulação central e os vasos que irrigam os músculos se contraem. 
Tabela 3. Amplitude térmica das temperaturas de superfície e garupa dos equinos antes e após o treino.

\begin{tabular}{lcc}
\hline \multicolumn{1}{c}{ Região/Padrão } & \multicolumn{2}{c}{ Amplitude Térmica $\left({ }^{\circ} \mathrm{C}\right)$} \\
\hline & Antes do Treino & Após o Treino \\
Cabeça/Branco & $5,2^{\mathrm{b}}$ & $7,5^{\mathrm{a}}$ \\
Cabeça/Vermelho & 6,0 & 7,5 \\
Cabeça/Amarelo & 0,8 & 1,5 \\
Traseira/Branco & $2,1^{\mathrm{a}}$ & $0,5^{\mathrm{b}}$ \\
Traseira/Vermelho & 0,1 & 0,2 \\
Traseira/Amarelo & $3,9^{\mathrm{a}}$ & $0,1^{\mathrm{b}}$ \\
\hline ab Diferentes letras na mesma linha indicam diferença estatística $(P<0,01)$.
\end{tabular}

Figura 3. Temperaturas (média, mínima e máxima) e amplitude térmica da cabeça dos equinos antes e após o treino, levando-se em consideração os diferentes padrões.

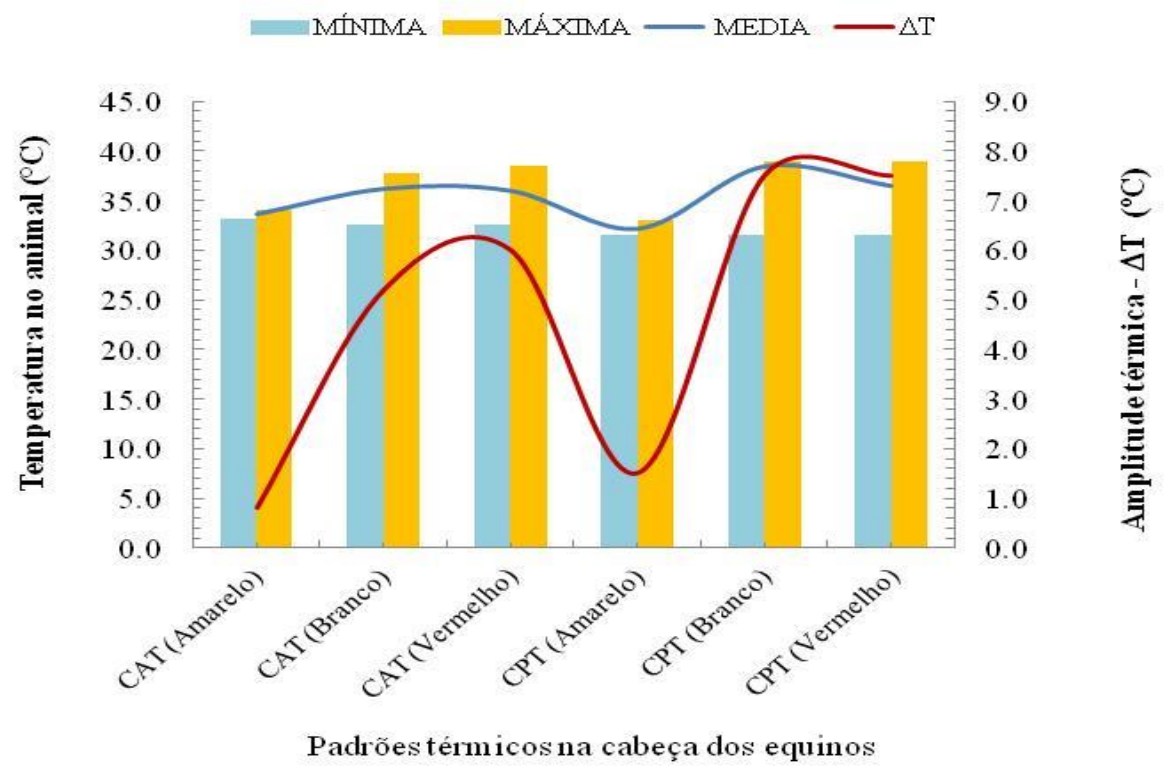

CAT: Cabeça antes do treino; CPT: Cabeça pós treino 
Figura 4. Temperaturas (média, mínima e máxima) e amplitude térmica da garupa dos equinos antes e após o treino, levando-se em consideração os diferentes padrões.

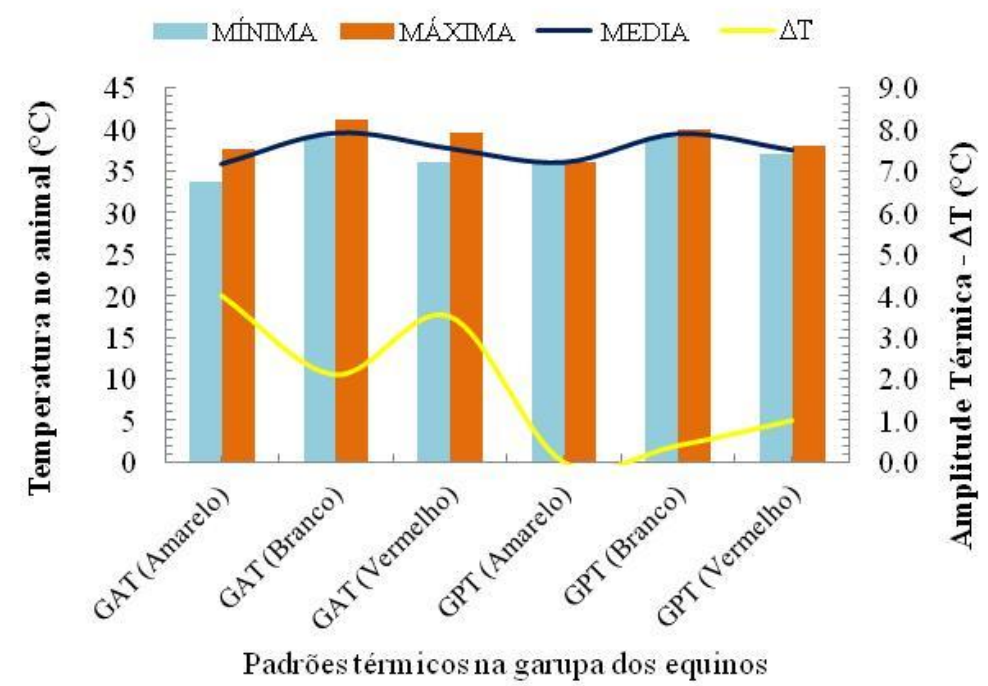

GAT: Garupa antes do treino; GPT: Garupa pós treino

Com relação aos parâmetros ocorrem sudorese e vasodilatação fisiológicos foi possível observar que a periférica, seguida por aumento da FC FR e a FC aumentaram $(P<0,05)$ após o e posterior elevação da FR (MARAl; treino (Tabela 4), ultrapassando os HAEEB 2010).

níveis basais de 8 a 16 mov min $^{-1}$ e 32 a

As variações térmicas nas superfícies 44 bat $\mathrm{min}^{-1}$, de acordo com corporais dos equinos refletem nas Cunningham (1999). A elevação dos frequências respiratórias com 70\% de parâmetros fisiológicos avaliados confiança, descritas por uma equação provavelmente relaciona-se com o ITU polinomial de segunda ordem, de 79,2 observado no estudo. Em conforme a equação 3. ambiente térmico desfavorável 
Tabela 4. Frequência respiratória (FR) e frequência cardíaca (FC) dos equinos antes e após o treino.

\begin{tabular}{lcc}
\hline Parâmetros Fisiológicos & \multicolumn{2}{c}{ Treino } \\
\hline & Antes do Treino & Após o Treino \\
FR $\left({\left.\text { mov } \text { min }^{-1}\right)}^{\text {FC }\left(\text { bat } \text { min }^{-1}\right)}\right.$ & $25,7 \pm 10,1^{\mathrm{b}}$ & $79,0 \pm 15,0^{\mathrm{a}}$ \\
\hline
\end{tabular}

ab Diferentes letras na mesma linha indicam diferença estatística $(P<0,05)$.

$$
F r=-49,547(\Delta T)^{\wedge} 2+127,59(\Delta T)+4,8903
$$

em que $F r$ é a frequência respiratória $\left(\right.$ mov $\left.\mathrm{min}^{-1}\right)$ e $\Delta T$ Ur é a amplitude térmica $\left({ }^{\circ} \mathrm{C}\right)$.

Ao analisar a primeira derivada na equação, verificou-se que a máxima amplitude térmica que os animais suportariam seria $1,2^{\circ} \mathrm{C}$, mas foi observado durante o período de monitoramento que o animal com · T de $1,8^{\circ} \mathrm{C}$ apresentou variações significativas nos parâmetros fisiológicos analisados, indicando que os mecanismos termoregulatórios foram acionados.

A amplitude térmica é uma variável resposta eficiente na avaliação de oscilações de temperatura de superfície e de parâmetros fisiológicos em equinos submetidos a esforço físico excessivo.
Um modelo polinomial explica as variações em frequência respiratória de equinos em treinamento intenso e pode ser utilizado para prognosticar o grau de estresse a partir das amplitudes térmicas.

\section{AGRADECIMENTOS}

A Coordenação de Aperfeiçoamento de Pessoal de Nível Superior (CAPES) pela concessão de bolsa de pós-doutorado à primeira autora. A Empresa Brasileira de Pesquisa Agropecuária (EMBRAPA) pela oportunidade de uso nesta pesquisa do termógrafo científico (Projeto MP5 PECUS/INFRA CNPq). 


\section{REFERÊNCIAS}

AGGARWAL, A.; SINGH, M. Changes in skin and rectal temperature in lactating buffaloes provided with showers and wallowing during hot-dry season. Tropical Animal Health Production, v.40, p.223-228, 2008.

AZEVEDO, M.; PIRES, M. F. A.; SATURNINO, H. M.; LANA, A. M. Q.; SAMPAIO, I.B.M.; MONTEIRO, J. B. N.; MORATO. L. E. Estimativa de níveis críticos superiores do índice de temperatura e umidade para vacas leiteiras 1/2, 3/4, 7/8 Holandês-Zebu em lactação. Revista Brasileira de Zootecnia, v. 34, p. 2000-2008, 2005.

BAÊTA, F. C:; SOUZA, C. F. Ambiência em edificações rurais conforto térmico. Viçosa, UFV. Universidade de Viçosa. 1997. 246p.

BOUZIDA, $\quad$ N.; BENDADA, A.; MALDAGUE, X. P. Visualization of body thermoregulation by infrared imaging. Journal of Thermal Biology, v. 34, p. 120-126, 2009.

CARVALHO, T.; MARA, L. S. Hidratação e nutrição no esporte. Revista Brasileira de Medicina do Esporte, v. 16, p.33-40, 2010.

CUNNINGHAM, J. G. Tratado de Fisiologia Veterinária. São Paulo: Guanabara Koogan, 1999, 528 p.

FILHO, J. C. M.; GOBESSO, A. A. O.; ETCHICHURY, M.; TITTO, C. G. Concentração de eletrólitos em equinos submetidos a diferentes temperaturas. Revista Brasileira de Saúde e Produção Animal, v. 10, p.236244, 2009.

IBGE. Instituto Brasileiro de Geográfico e Estatístico. Prod. Pec. munic., Rio de Janeiro, v. 40, p. 1 - 71, 2012.

JONES, S. Horsback riding in the dog days. Animal Science e-News, v. 2, p.34, 2009.

KANEPS. R. J. Equine exercise physiology: the science of exercise in the athletic horse. Philadelphia: Elsevier, 2008, p. 382-386.

KOTRBA, R.; KNÍZKOVÁ, I.; KUNC, P.; BARTOS, L. Comparison between the coat temperature of the eland and dairy cattle by infrared thermography. Journal of Thermal Biology, v. 32, p. 355-359, 2007.

MARAI, I.F.M.; HAEEB, A.A.M. Buffalo's biological functions as affected by heat stress: a review. Livest Science, v. 127, p. 89-109, 2010.

MARLIN, D. J.; SCOTT, C. M.; ROBERTS, C.A.; CASAS, I.; HOLAH, G.; SCHROTER, R.C. Post exercise changes in compartmental body temperature accompanying intermittent cold water cooling in the hyperthermic horse. Equine Veterinary Journal, v. 30, p. 28-34, 1998.

MARTORANO, L.G.; PEREIRA, L.C.; CEZAR, E. G. M.; PEREIRA, I.C.B. Estudos climáticos do Estado do Pará, classificação climática (Kõppen) e 
deficiência hídrica. Belém, SUDAM/EMBRAPA, SNLCS, p. 53, 1993.

MARTORANO, L.G.; VITORINO, M. I.; DA SILVA, B. P. P. C.; DE MORAES, J. R. S. C.; LISBOA, L. S.; SOTTA, E. D.; REICHARDT, K. Climate conditions in the eastern amazon: Rainfall ariability in Belem and indicative of soil water deficit. African Journal of Agricultural Research, v. 12, p. 1801-1810, 2017.

MENEGASSI, S. R. O.; BARCELLOS, J. O. J.; DIAS, E. A.; KOETZ J. R. C.; PEREIRA, G. R.; PERIPOLLI, V.; MCMANUS, C.; CANOZZI, M. E. A.; LOPES, F. G. Scrotal infrared digital thermography as a predictor of seasonal effects on sperm traits in Braford bulls. International Journal of Biometeorology, v. 59, p. 357-364, 2015.

MCCONAGHY, F. F.; HODGSON, D. R.; ROSE, R. J.; HALES, J. R. S. Redistribution of cardiac output in response to heat exposure in the pony. Equine Veterinary Journal Supplement, v. 22, p. 42-46, 1996.

MOURA, D. J.; MATARAZZO, S. V.; SILVA, I. J. O.; LIMA, K. A. O. Efeito da utilização de sistemas de climatização nos parâmetros fisiológicos do gado leiteiro. Engenharia Agrícola, v. 26, p.663-671, 2006.

MCCUTCHEON, L. J.; GEOR, R. J. SILVA, J. A. R; ARAÚJO, A.A.; LOURENÇO JUNIOR, J. B.; SANTOS, N. F. A.; GARCIA, A. R.; NAHÚM, B. S. Conforto térmico de búfalas em sistema silvipastoril na Amazônia Oriental.
Pesquisa Agropecuária Brasileira, v. 46, p. 1364-1371, 2011.

PERISSINOTTO, M.; MOURA, D. J.; MATARAZZO, S. V.; SILVA, I. J. O.; LIMA, K. A. O. Efeito da utilização de sistemas de climatização nos parâmetros fisiológicos do gado leiteiro. Engenharia Agrícola, v. 26, p. 663-671, 2006.

SOUZA, B. B.; SOUZA, E. D.; CEZAR, M. $F$. et al. Temperatura superficial e índice de tolerância ao calor de caprinos de diferentes grupos raciais no semi-árido nordestino. Ciência e Agrotecnologia. v. 32, p. 275-280, 2008.

SYKES, D. J.; COUVILLIONA, J. S.; CROMIAKA, A.; BOWERS. S.; SCHENCKA, E.; CRENSHAWA, M.; RYANA, P. L. The use of digital infrared thermal imaging to detect estrus in gilts. Theriogenology, v. 78, p.147-152, 2012.

TITTO, E. A. L.; PEREIRA, A. M. F.; TOLEDO, L. R. A.; PASSINI, R.; NOGUEIRA FILHO, J. C. M.; GOBESSO, A. A. O.; ETCHICHURY, M.; TITTO, C. G. Concentração de eletrólitos em equinos submetidos a diferentes temperaturas. Revista Brasileira de Saúde e Produção Animal, v. 10, p. 236244, 2009.

THOM, E. C. The discomfort index. Weatherwise, v. 12, n. 7, p. 57-59, 1959.

WILSON, A.; WELLER, R. The Biomechanics of the Equine Limb and Its Effect on Lameness. In: Ross MW, Dyson S, editors. Diagnosis and 
Management of Lameness in the Horse Saint Louis: W.B. Saunders, 2011. p. 270-281.

ZOTTI, C. A. Desempenho, respostas fisiológicas e comportamentais de novilhas leiteiras mantidas em diferentes regimes de ventilação forçada. 2010. 63f. Dissertação (Mestrado em Zootecnia) - Instituto de Zootecnia - APTA/SAA, Nova Odessa, 2010. 